Portland State University

PDXScholar

Electrical and Computer Engineering Faculty

Publications and Presentations

Electrical and Computer Engineering

$3-15-2013$

\title{
Depth-Based Signal Separation with Vertical Line Arrays in the Deep Ocean
}

\author{
Reid McCargar \\ John Hopkins University, reid.mccargar@jhuapl.edu
}

Lisa M. Zurk

Portland State University, zurkl@pdx.edu

Follow this and additional works at: https://pdxscholar.library.pdx.edu/ece_fac

Part of the Electrical and Computer Engineering Commons

Let us know how access to this document benefits you.

\section{Citation Details}

McCargar, Reid, and Lisa M. Zurk. "Depth-based signal separation with vertical line arrays in the deep ocean." The Journal of the Acoustical Society of America 133, no. 4 (2013): EL320-EL325.

This Article is brought to you for free and open access. It has been accepted for inclusion in Electrical and Computer Engineering Faculty Publications and Presentations by an authorized administrator of PDXScholar. Please contact us if we can make this document more accessible: pdxscholar@pdx.edu. 


\title{
Depth-based signal separation with vertical line arrays in the deep ocean
}

\author{
Reid McCargar and Lisa M. Zurk \\ Northwest Electromagnetics and Acoustics Research Laboratory (NEAR-Lab), \\ Department of Electrical and Computer Engineering, Portland State University, Portland, \\ Oregon 97201 \\ rmccar@cecs.pdx.edu,zurkl@pdx.edu
}

\begin{abstract}
Deep vertical line arrays can exploit the reliable acoustic path (RAP), which provides low transmission loss (TL) for targets at moderate ranges, and increased TL for distant interferers. However, nearby surface interference also has favorable RAP propagation and cannot be separated from a submerged target without horizontal aperture. In this work, a physics-based Fourier transform variant is introduced, which achieves depth-based signal separation by exploiting the spatial structure resulting from the coherent addition of the direct and surface-reflected propagation paths present for submerged sources. Simulation results demonstrate depth-based signal separation without requiring knowledge of the ocean environment.
\end{abstract}

(C) 2013 Acoustical Society of America

PACS numbers: 43.60.Uv, 43.30.Wi, 43.60.Mn [DC]

Date Received: December 15, 2012 Date Accepted: February 27, 2013

\section{Introduction}

Using data from the Northeast Pacific, Gaul et al. ${ }^{1}$ demonstrated that a sensor deployed below the critical depth (the depth below the sound speed minimum at which the sound speed equals the speed at the surface) can exploit deep-ocean propagation attributes to achieve 10-25 dB lower received levels from distant sound sources than receivers above the critical depth. These observations were supported in a simulation study by Li et al., ${ }^{2}$ and attributed to the deep sound channel confining low-loss propagation paths to above the critical depth, below which bottom losses become significant.

Sound can reach a deep receiver from sources at moderate ranges $(20-35 \mathrm{~km})$ via the reliable acoustic path (RAP), ${ }^{3}$ a direct and possibly a surface-reflected pathway with no bottom interaction or refractive turning points. Accordingly, predicted received levels are 10-20 dB higher than for a shallow receiver at the same range, which relies on environmentally sensitive bottom reflections, surface ducts, or upward-refracted convergence zone arrivals.

A deep vertical line array (VLA) can leverage the steep vertical angles and low loss of RAP arrivals from nearby targets to discriminate them from distant shipping noise, which experiences increased transmission loss (TL) and arrives near horizontal. However, nearby interferers also experience favorable RAP propagation and arrive at steep angles, presenting a challenge for a VLA which lacks azimuthal rejection. Historically, this challenge has been addressed with adaptive array processing techniques and matched-field approaches ${ }^{4,5}$ but many of these techniques are susceptible to environmental uncertainty. It is shown here that additional separation of surface vs submerged sources can be achieved by exploiting Lloyd's mirror pattern, which exists due to the coherent summation of the direct and surface-reflected sound. This pattern can be described with relatively simple expressions, and exploited to provide depth-based signal separation (DBSS).

Signal separation is accomplished by applying a physics-based Fourier transform variant to the power output of a plane-wave beamformer as it evolves over time. The technique is derived here using image-theory, then tested on synthetic deep-VLA 
data, generated using a rigorous acoustical model to simulate a moving target, nearby moving interferers, wind noise, and distant shipping noise.

\section{DBSS theory and technique}

Figure 1(a) illustrates the image-theory geometry, indicating the paths of the direct and surface-reflected components of the acoustic field from a submerged point source at constant depth $z_{\mathrm{s}}$, and range $r\left(t_{l}\right)$, with spectral amplitude $S(\omega)$. The source moves in a water column with sound speed $c$, with an $r$-z-plane velocity component $v_{s r}\left(t_{l}\right)$.

Although image theory does not accurately model propagation in a refracting environment, it is used here to gain insight, and to develop expressions describing the robust interaction of the direct and surface-reflected propagation paths. The imagetheory expressions are applied later to synthetic deep VLA data, generated using KRAKEN and OASES-OASN. ${ }^{6}$

The field sampled during the $l$ th snapshot is received by an $N$-element VLA with uniform receiver separation, $\delta$, and midpoint depth, $\bar{z}$. In the far field, the addition of the two image-theory field components,

$$
\begin{aligned}
p\left(r\left(t_{l}\right), z_{n}, \omega\right) & =S(\omega)\left[\frac{e^{i k R_{n}^{+}\left(t_{l}\right)}}{R_{n}^{+}\left(t_{l}\right)}-\frac{e^{i k R_{n}^{-}\left(t_{l}\right)}}{R_{n}^{-}\left(t_{l}\right)}\right] \\
& \approx-2 i S(\omega) \frac{\sin \theta\left(t_{l}\right)}{\bar{z}} e^{i\left[k\left(\bar{z} / \sin \theta\left(t_{l}\right)\right)\right]} \sin \left(k z_{\mathrm{s}} \sin \theta\left(t_{l}\right)\right) e^{i k(n-N / 2) \delta \sin \theta\left(t_{l}\right)},
\end{aligned}
$$

describes a plane wave incident at vertical angle $\theta$, where $k=\omega / c$, and $n$ is the receiver index. The $\sin \left(k z_{\mathrm{s}} \sin \theta\left(t_{l}\right)\right)$ factor causes Lloyd's-mirror interference fringes which increase in spatial frequency as a function of source depth.

Figures 1(b) and 1(c) show TL at $150 \mathrm{~Hz}$ as a function of range and depth for sources at $z_{\mathrm{s}}=7 \mathrm{~m} \mathrm{[1(b)]} \mathrm{and} z_{\mathrm{s}}=50 \mathrm{~m}$ [1(c)], predicted with KRAKEN for a $5000-\mathrm{m}$ water column with a Munk sound speed profile and a 100-m silt sediment layer over a semi-infinite basalt basement. ${ }^{6}$ Although refractive bending and multiple boundary reflections are apparent, the depth-dependent interference structure predicted by imagetheory is a dominant and distinguishing feature of the two TL plots, with the shallow source showing lower frequency spatial interference than the deeper source.

Synthetic snapshot data were generated for a deep VLA with $N=16$, $\delta=5 \mathrm{~m}$, and $\bar{z}=4950 \mathrm{~m}$ in this geoacoustic environment. Target and distant interference signals were computed at $150 \mathrm{~Hz}$ with KRAKEN for the parameters in Table 1, and wind noise realizations were drawn from the wind noise covariance, which was computed with OASES-OASN for $55 \mathrm{~dB}$ wind noise.

A data covariance was computed using 5-s snapshots and a recursive averaging technique which incurred the same average SNR loss as averaging $2 N-3$ snapshot covariances. ${ }^{4,7}$ Minimum variance distortionless response steering vectors were computed

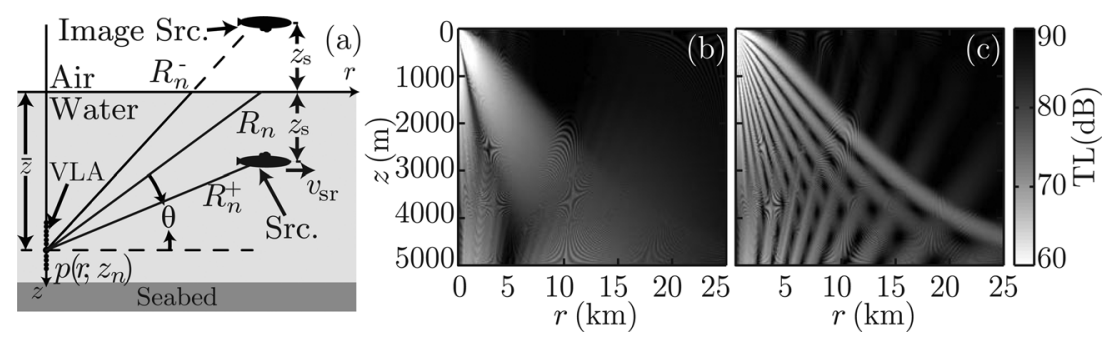

Fig. 1. Panel (a) shows an illustration of the image-theory geometry, with time-dependence implied for a source in motion. Plots in (b) and (c) show TL predicted for a Munk sound speed profile, using KRAKEN, for a source at (b) $z_{\mathrm{s}}=7 \mathrm{~m}$, showing low-spatial-frequency interference structure, and (c) $z_{\mathrm{s}}=50 \mathrm{~m}$, showing high-spatial-frequency Lloyd's-mirror-type structure. Plots in (b) and (c) share a common depth axis. 
Table 1. Source parameters used to generate synthetic data. Constant speed, depth, and heading source trajectories were parameterized by range and time at closest point of approach (CPA). Distant interferers were considered stationary, with ranges and depths sampled uniformly from the intervals given.

\begin{tabular}{lccc}
\hline \hline & $\begin{array}{c}\text { Nearby interferers } \\
\text { (Fig. 3) only }\end{array}$ & Distant interferers & Target \\
\hline$N_{\text {sources }}$ & 3 & 50 & 1 \\
CPA range (km) & $8,15,18$ & $100-1500$ & 2 \\
CPA time (min) & $-10,-45,150$ & - & 45 \\
Speed (m/s) & 13 & -10 & 5 \\
Depth (m) & 7 & & 7 [Fig. 2(a)] 50 [Fig. 2(b) \\
$150 \mathrm{~Hz}$ source & 160 & 160 & and Fig. 3] \\
level $\left(\mathrm{dB} \mathrm{re} 1 \mu \mathrm{Pa}^{2} / \mathrm{Hz}\right)$ & & & 110 \\
\hline \hline
\end{tabular}

with a $3-\mathrm{dB}$ white noise gain constraint, and used to beamform the synthetic data. ${ }^{8}$ Vertical time records (VTRs) which show the beamformed signal power, $P\left(\omega, \sin \theta, t_{l}\right)$, as a function of steering angle and snapshot time are shown for targets at $z_{\mathrm{s}}=7 \mathrm{~m}$ [Fig. 2(a)] and $z_{\mathrm{s}}=50 \mathrm{~m}$ [Fig. 2(b)].

Image theory predicts a depth-dependent factor in the $n, m$ th element of the instantaneous covariance matrix,

$$
K_{n m}\left(\omega, t_{l}\right)=2\left(\frac{S(\omega) \sin \theta\left(t_{l}\right)}{\bar{z}}\right)^{2}\left[1-\cos \left(2 k z_{\mathrm{s}} \sin \theta\left(t_{l}\right)\right)\right] e^{i k(n-m) \delta \sin \theta\left(t_{l}\right)} .
$$

The low-frequency fluctuations apparent beneath the dashed blue trace in the VTR for the shallow target [Fig. 2(a)], and higher-frequency fluctuations apparent beneath the dashed red trace for the deeper target [Fig. 2(b)], are in qualitative agreement with this expression, which forms the basis for the DBSS technique.

The sinusoidal form of the depth-dependent modulation in Eq. (2) suggests that DBSS could be achieved by Fourier analysis of the signal power along the vertical-angle trajectory of the target; however, snapshots are collected uniformly in time, while the sinusoidal fluctuations predicted by image theory vary harmonically with the sine of the elevation angle, which is generally a simple but nonlinear function of time. To match the structure of the depth-dependent signal, the conventional Fourier integral is reformulated as a path integral of the beamformed signal power along the integration path, $P\left(\omega, \sin \theta_{\mathrm{tr}}\left(t_{l}\right)\right)$, with the kernel modified so that the complex phase matches the argument of the sinusoidal depth-dependent modulation factor in Eq. (2),
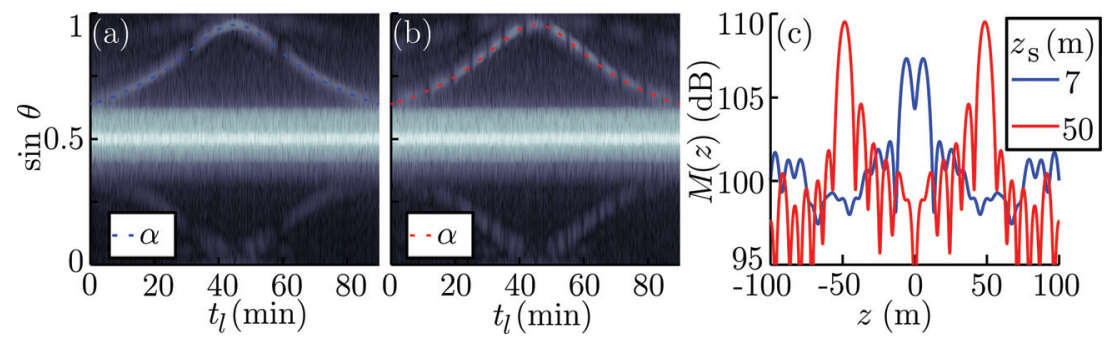

Fig. 2. Beam output shown as a VTR at $150 \mathrm{~Hz}$, plotted with $80 \mathrm{~dB}$ (re peak) dynamic range and a common $y$-axis in (a) and (b), with overlaid integration paths, $\alpha$, shown as dashed lines. Synthetic data were generated using KRAKEN for a source at (a) $z_{\mathrm{s}}=7 \mathrm{~m}$ and (b) $z_{\mathrm{s}}=50 \mathrm{~m}$, and 50 distant interferers. 55-dB wind noise was simulated with OASES. The plot in (c) shows the physics-based DBSS transform output, $M(z)$, applied to the overlaid traces in (a) and (b) after re-normalization and de-meaning. 


$$
M(z, \omega)=\int_{\alpha} P\left(\omega, \sin \theta_{\mathrm{tr}}\left(t_{l}\right)\right) e^{-i 2 k z \sin \theta_{\mathrm{tr}}\left(t_{l}\right)}\left|d \sin \theta_{\mathrm{tr}}\left(t_{l}\right)\right| .
$$

The angular trajectory, $\sin \theta_{\mathrm{tr}}\left(t_{l}\right)$, describes a hypothesis for the time-dependence of a candidate target's elevation angle, and dictates the integration path, $\alpha$. The angular trajectories which were used to compute the DBSS result in Fig. 2(c), are shown as dashed traces overlaying the VTRs in Figs. 2(a) and 2(b).

Assuming the data covariance takes the form of Eq. (2), and the track hypothesis matches the actual angular trajectory of the target, the DBSS result predicted by image theory can be expressed as a convolution, denoted by $\otimes$ :

$$
\begin{aligned}
M(z, \omega)= & 2 \frac{S^{2}(\omega)}{\bar{z}^{2}} \int_{\sin \theta_{\mathrm{tr}}\left(t_{1}\right)}^{\sin \theta_{\mathrm{tr}}\left(t_{L}\right)} \sin ^{2} \theta_{\mathrm{tr}}\left(t_{l}\right) e^{-i 2 k z \sin \theta_{\mathrm{tr}}\left(t_{l}\right)}\left|\frac{d \sin \theta_{\mathrm{tr}}\left(t_{l}\right)}{d t_{l}}\right| d t_{l} \\
& \otimes \frac{\pi}{k}\left[\delta(z)-\frac{1}{2} \delta\left(z \pm z_{\mathrm{s}}\right)\right]
\end{aligned}
$$

where a change of variables has converted the path integral to a time integration.

The integral of the depth-independent factors is well-localized and easily solvable. This important result predicts that the transform will have depth-dependent peaks at $z= \pm z_{\mathrm{s}}$, which means each integration path can be associated with a source depth, thereby achieving depth-based signal separation. Assuming the array depth is approximately known, the signal power along the analysis path can be re-normalized and de-meaned prior to transforming in order to estimate the source spectrum level, $S(\omega)$, and to remove the peak at $z=0$.

On discrete snapshot data, Eq. (3) is implemented as a summation,

$$
M(z, \omega)=\sum_{l=1}^{L} P\left(\omega, \sin \theta_{\operatorname{tr}}\left(t_{l}\right)\right) e^{-i 2 k z \sin \theta_{\mathrm{tr}}\left(t_{l}\right)}\left|\Delta \sin \theta_{\mathrm{tr}}\left(t_{l}\right)\right|,
$$

where $\Delta \sin \theta_{\mathrm{tr}}\left(t_{l}\right)=\sin \theta_{\mathrm{tr}}\left(t_{l}\right)-\sin \theta_{\mathrm{tr}}\left(t_{l-1}\right)$ is the discrete approximation to the continuous-time derivative of the elevation angle along the analysis path.

After de-meaning and re-normalization, the discrete DBSS transform was applied along the traces in Fig. 2(a) and 2(b) to produce the transform result shown in Fig. 2(c). No environmental knowledge was necessary, and the transform was computed assuming an isovelocity channel with $c=1500 \mathrm{~m} / \mathrm{s}$. Unambiguous peaks are apparent at the true depth of both contacts, in excellent agreement with the imagetheory prediction.

\section{Simulation results}

The result in Fig. 2(c) demonstrates the robustness of the DBSS technique to unknown geoacoustic parameters, wind noise, and near-horizontally incident distant interference. Nearby interference, which arrives at steep elevation angles and experiences favorable RAP propagation, poses the most significant challenge for deep VLAs. In this section, a more challenging environment with dynamic nearby interference is considered by adding nearby moving interferers, described in Table 1, to the synthetic data from Fig. 2(c). Figure 3(a) shows the simulated VTR, in which the target signal is briefly discernible from the strong interference.

A sonar operator must attempt to determine which of the signals in the VTR correspond to contacts of interest, which is typically accomplished by performing spectral classification along an analysis trace (scissor-gram analysis). Trace selection can be accomplished by a detection and tracking algorithm, track-before-detect filter bank, or by an operator's visual inspection of the broadband or sub-band VTR. Here, candidate traces were identified in Figs. 2 and 3 by visual inspection, one corresponding to the 

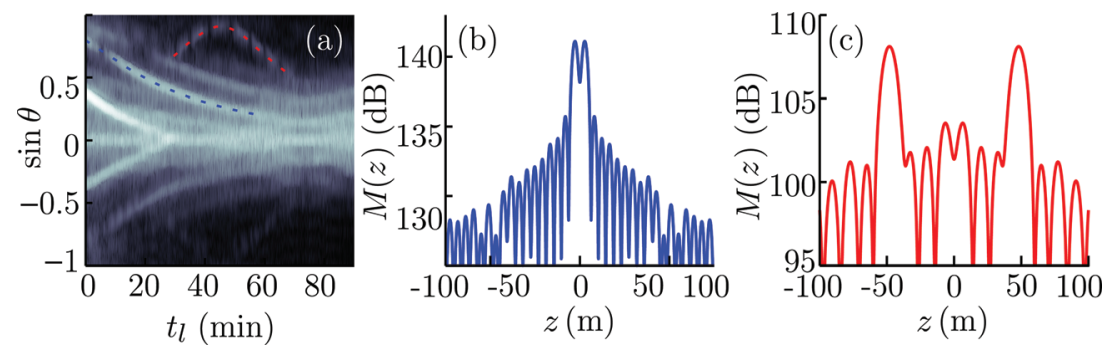

Fig. 3. The plot in (a) shows the simulated VTR with overlaid traces for the $z_{\mathrm{s}}=7 \mathrm{~m}$ interferer multipath (blue), and $z_{\mathrm{s}}=50 \mathrm{~m}$ target (red), plotted with $80 \mathrm{~dB}$ dynamic range (re peak). The plot in (b) shows the nearby interferer multipath-trace DBSS transform (near-surface source). The plot in (c) shows the target trace DBSS transform (submerged source).

target at $z_{\mathrm{s}}=50 \mathrm{~m}$ (red), and the other to a multipath arrival from the nearest interferer at $z_{\mathrm{s}}=7 \mathrm{~m}$ (blue), and classification was achieved with the new DBSS technique.

The power output along the contact traces was re-normalized and de-meaned before applying the DBSS transform. Figure 3(b) shows the transform output for the nearby interferer multipath, and Fig. 3(c) shows the result for the submerged target.

Depth-dependent peaks in Figs. 3(b) and 3(c) remain well-resolved, in close agreement with the true depths, and unambiguous against the background. Depthdependent peak width and angular extent of the analysis trace are coupled through an uncertainty principle, which causes the peak broadening apparent in Fig. 3(c), compared to Fig. 2(c), as well as decreased processing gain. The apparent improvement in peak resolution for the shallow interferer in Fig. 3(b) can be attributed to the received level from the interferer multipath being $\approx 30 \mathrm{~dB}$ higher than from the target, and considerably higher than wind noise and distant shipping levels. The minimal deviation of peak locations from the true depth illustrates the robustness of the DBSS technique to an unknown environment, particularly for the nearby interferer multipath, which experiences three passes through the refracting water column, and multiple boundary interactions.

\section{Conclusion}

This paper has described a technique to achieve depth-based signal separation using a modified Fourier transform, derived using simple image-theory expressions to describe the interaction of the direct and surface-reflected sound from a submerged contact. This approach was plausible due to the practically ubiquitous strong reflection from the sea surface, and the insensitivity of the reliable acoustic path to propagation conditions. In simulations incorporating representative geoacoustic parameters and dynamic interference, the DBSS technique achieved accurate depth estimation, and 6-10 dB unambiguous depth-based separation using no environmental compensation in the processing.

Doppler effects, spatially extended sources (rather than point sources), internal waves, and surface swell were not included in this analysis, but are expected to have little influence on processing performance at the low frequencies commonly used for passive detection.

\section{Acknowledgment}

This work was supported by the Office of Naval Research, ONR-321, Signal Processing.

\section{References and links}

${ }^{1}$ R. Gaul, D. Knobles, J. Shooter, and A. Wittenborn, “Ambient noise analysis of deep-ocean measurements in the Northeast Pacific,” IEEE J. Ocean. Eng. 32, 497-512 (2007). 
${ }^{2} \mathrm{Z}$. Li, L. M. Zurk, and B. Ma, "Vertical arrival structure of shipping noise in deep water channels," in OCEANS 2010 (2010), pp. 1-8.

${ }^{3}$ R. J. Urick, Principles of Underwater Sound (McGraw-Hill, New York, 1983).

${ }^{4}$ A. Baggeroer, W. Kuperman, and P. Mikhalevsky, "An overview of matched field methods in ocean acoustics," IEEE J. Ocean. Eng. 18, 401-424 (1993).

${ }^{5}$ L. M. Zurk, N. Lee, and J. Ward, "Source motion mitigation for adaptive matched field processing,"

J. Acoust. Soc. Am. 113, 2719 (2003).

${ }^{6}$ F. B. Jensen, W. A. Kuperman, M. B. Porter, and H. Schmidt, Computational Ocean Acoustics (Springer, New York, 2000).

${ }^{7}$ H. Van Trees, Optimum Array Processing (Wiley, New York, 2002).

${ }^{8}$ H. Cox, R. Zeskind, and M. Owen, "Robust adaptive beamforming," IEEE Audio, Speech, Lang. Process. 35, 1365-1376 (1987). 